\title{
Response to Comment on: the Use of Visceral Adiposity Index to Predict Diabetes Remission in Low BMI Chinese Patients after Bariatric Surgery
}

\author{
${\text { Zhigang } \mathrm{Ke}^{1}(\mathrm{D}) \cdot \text { Weidong Tong }}^{1}$
}

Received: 19 December 2020 / Revised: 19 December 2020 / Accepted: 22 December 2020 / Published online: 3 January 2021

(C) The Author(s), under exclusive licence to Springer Science+Business Media, LLC part of Springer Nature 2021

We would like to thank the authors for their thoughtful insights and comments made to our recent publication "the use of visceral adiposity index to predict diabetes remission in low BMI Chinese patients after Bariatric surgery" [1].

Adipose tissue is a complex endocrine organ, involved not only in fat storage but also in releasing several adipokines, which could be critically involved in development of insulin resistance, type 2 diabetes mellitus (T2DM), metabolic syndrome, and cardiovascular disease [2]. The visceral adiposity index (VAI) has been currently considered to be a reliable indicator of visceral adipose tissue mass and visceral adipose dysfunction, and has been found to be related to insulin resistance and cardiovascular disease risk [3], which may be attributed to the role of adipokines. We agree that all the known predictors associated with T2DM remission should be included in a regression model, such as age, diabetes duration, body mass index (BMI), visceral fat area, subcutaneous fat area, fasting C-peptide, $\mathrm{HbAlc}$, insulin use, and other medications used [4-6]. These predictors can reflect the patient's functional B-cell reserve, thereby measuring the severity of T2DM. Percent excess weight loss (\%EWL) is another predictor of T2DM remission post-surgically [7], which was not included in our study as a predictor because it is not a preoperative predictor.

As we all know, both CT and MRI can easily distinguish and quantify subcutaneous fat and visceral fat, and can also accurately quantify liver fat, but the relatively higher medical cost, time-consuming and potential radiation exposure defects prevent its wide clinical practice. VAI is a good indicator for easy measurement and evaluation of visceral fat mass. However, to evaluate more accurately and refine the

Weidong Tong

vdtong@163.com

1 Department of General Surgery, Daping Hospital, Army Medical University, Chongqing 400042, China stratification of abdominal obesity, the addition of CT or MRI may be useful in future studies.

As the author mentioned, the scientific evidence of the past years on the use of VAI has allowed us realize the usefulness and limitations of VAI. Further research on obesity classification and disease stratification are needed to better verify the usefulness of VAI.

\section{Compliance with Ethical Standards}

Conflict of Interest The authors declare that they have no conflicts of interest.

\section{References}

1. Ke Z, Li F, Gao Y, et al. The use of visceral adiposity index to predict diabetes remission in low BMI Chinese patients after bariatric surgery. Obes Surg. 2020;

2. Recinella L, Orlando G, Ferrante C, et al. Adipokines: new potential therapeutic target for obesity and metabolic, rheumatic, and cardiovascular diseases. Front Physiol. 2020;11:578966.

3. Amato MC, Giordano C, Galia M, et al. Visceral adiposity index: a reliable indicator of visceral fat function associated with cardiometabolic risk. Diabetes Care. 2010;33(4):920-2.

4. Wang GF, Yan YX, Xu N, et al. Predictive factors of type 2 diabetes mellitus remission following bariatric surgery: a meta-analysis. Obes Surg. 2015;25(2):199-208.

5. Lee WJ, Chong K, Chen SC, et al. Preoperative prediction of type 2 diabetes remission after gastric bypass surgery: a comparison of DiaRem scores and ABCD scores. Obes Surg. 2016;26(10):241824.

6. Yu HY, Di JZ, Bao YQ, et al. Visceral fat area as a new predictor of short-term diabetes remission after Roux-en-Y gastric bypass surgery in Chinese patients with a body mass index less than $35 \mathrm{~kg} /$ $\mathrm{m}(2)$. Surg Obes Relat Dis. 2015;11(1):6-13.

7. Hamza N, Abbas MH, Darwish A, et al. Predictors of remission of type 2 diabetes mellitus after laparoscopic gastric banding and bypass. Surg Obes Relat Dis. 2011;7(6):691-6.

Publisher's Note Springer Nature remains neutral with regard to jurisdictional claims in published maps and institutional affiliations. 